\title{
Concurrent finite element simulation of quadrupolar and dipolar flow noise in low Mach number aeroacoustics
}

\author{
Oriol Guasch ${ }^{\mathrm{a}}$, Arnau Pont ${ }^{\mathrm{b}}$, Joan Baiges $^{\mathrm{b}}$, Ramon Codina $^{\mathrm{b}}$ \\ ${ }^{a}$ GTM - Grup de recerca en Tecnologies Mèdia, La Salle, Universitat Ramon Llull \\ C/ Quatre Camins 2, 08022 Barcelona, Catalonia \\ ${ }^{b}$ Universitat Politècnica de Catalunya, \\ Jordi Girona 1-3, Edifici C1, 08034 Barcelona, Catalonia
}

\begin{abstract}
The computation of flow-induced noise at low Mach numbers usually relies on a two-step hybrid methodolgy. In the first step, an incompressible fuid dynamics simulation (CFD) is performed and an acoustic source term is derived from it. The latter becomes the inhomegenous term for an acoustic wave equation, which is solved in the second step, often resorting to boundary integral formulations. In the presence of rigid bodies, Curle's acoustic analogy is probably the most extended approach. It has been shown that Curle's boundary dipolar noise contribution does in fact correspond to the diffraction of the quadrupolar aerodynamic noise generated by the flow past the rigid body. In this work, advantage is taken from this fact to propose an alternative computational methodology to get the individual quadrupolar and dipolar contributions to the total acoustic pressure. For any linear acoustic wave operator, the unknown acoustic pressure can be split into its incident and diffracted components and be computed simultaneously to the incompressible flow field, in a single finite element computational run. This circumvents the problem found in Curle's analogy of needing the total pressure at the body's boundary, which includes the acoustic pressure fluctuations. The latter cannot be obtained from an incompressible CFD simulation. The proposed unified strategy could be beneficial for a large variety problems such as those involving noise generated from duct terminations, or those related with the simulation of fricatives in numerical voice production, among many others.
\end{abstract}

Keywords: Computational aeroacoustics, Flow noise, quadrupolar noise, dipolar noise, diffraction

\section{Introduction}

In the direct noise computation (DNC) approach to aeroacoustics (see e.g., [1]), aerodynamic noise is straightforwardly obtained as a bypass product of the unsteady and/or turbulent flow motion, driven by the compressible Navier-Stokes equations. However, DNC is only feasible for academic cases, and a few industrial ones, due to its high computational cost. As a consequence, most computational aeroacoustics (CAA) strategies to date have relied on hybrid approaches [2], which can be applied whenever there is no important feedback from the acoustic field to the aerodynamic one. Hybrid approaches usually consist of a first finite element (FEM), or finite volume, computational fluid dynamics (CFD) simulation to obtain the aerodynamic velocity and pressure fields, from which some acoustic sources can be derived. The latter are set as the inhomogeneous term of a wave equation that is solved to get the acoustic pressure field in a second numerical simulation. The acoustic pressure is usually computed with the boundary element method (BEM) if the sound pressure is to be found at long distances from the source region, though FEM is also feasible if the computational domain is not too large $[3,4,5,6,7]$.

Email address: oguasch@salleurl.edu (Oriol Guasch)
URL: www.salle.url.edu/ oguasch (Oriol Guasch) 
In this work, focus is placed on aeroacoustics at low Mach numbers. The most popular option for unbounded flows probably is that of making use of Lighthill's acoustic analogy [8]. First, an incompressible CFD simulation is carried out and the double divergence of the Reynolds tensor is computed from it as an approximation to Lighthill's tensor (see $[9,10,11]$ for a detailed justification). The latter is convolved with the free-space Green function for the wave equation to get the acoustic pressure radiated by flow motion. This allows one to identify the problem of flow noise emission with that of sound radiation by a distribution of quadrupoles in free space. Though the most celebrated acoustic analogy is that of Lighthill, many other analogies have been derived. Some emphasize the role of vorticity in aerodynamic noise production [12, $13,14]$ while others attempt at transferring mean flow effects from Lighthill's source term to the wave operator (see e.g., [15] for a review and also [16]). Approaches standing on acoustic perturbation equations (e.g., $[17,18,19])$ derived from linearization of the Euler equations (e.g., $[11,20]$ ) are also quite common.

All the strategies in the precedent paragraph are intended for aerodynamic sound propagation in free field. To consider the effects of bodies within the flow on aerodynamic noise, one could proceed analogously to what is done for Lighthill's acoustic analogy, but convolving the source term with a tailored Green function that accounts for the body boundary conditions. The problem is that for complex geometries tailored Green functions are not available. Curle [21] proposed a way out to this difficulty by identifying the influence of rigid bodies with that of a distribution of surface dipoles. The free-space Green function can be used instead of the tailored one, though at the price of taking into account a new source of sound in the simulations. Curle's formulation was extended for non-rigid bodies in the well-known Ffowcs Williams-Hawkings equation [22].

Unfortunately, when applied to low Mach number aeroacoustics, Curle's formulation presents a problem. The dipole surface integral term involves the gradient of the total pressure in the normal direction to the boundary of the rigid body. The total pressure includes the acoustic fluctuations, which cannot be obtained from an incompressible CFD simulation. Recently, it has been proposed to surpass this difficulty by just considering the gradient of the incompressible flow pressure and by replacing the wave equation Green function with that of the Laplacian operator [23]. Another strategy will be followed hereafter. This is based on the observation that Curle's analogy is actually a sharp way to account for aerodynamic noise generated by surfaces, though what it is really happening, from a physical point of view, is that waves generated by flow motion get diffracted by the rigid body [24, 25, 26].

In this paper, we take advantage of this fact and propose a methodology for the simultaneous computation of the flow field, and of the turbulent and surface diffracted contributions to the total acoustic pressure field. At each time step of the simulation, the flow incompressible aerodynamic velocity and pressure are obtained by solving the incompressible Navier-Stokes equations, and input into a wave equation for the acoustic pressure, as if no rigid body was present. This computed acoustic pressure generated by the wake past the body has a quadrupolar character and plays the role of an incident pressure field on the rigid body boundary. Therefore, the incident pressure is used in turn to solve a wave equation for the acoustic pressure diffracted by the rigid body, at the same computational time step. In this way, the complete time evolution of the separate contributions to the acoustic pressure generated by flow motion (quadrupolar) and by surface diffraction (dipolar) is obtained at the end of the simulation. All the partial differential equations involved in this methodology have been solved by means of FEM, resorting to stabilization strategies when necessary. A preliminary, short version of this work can be found in [27].

The paper is organized as follows. The basic theory and problem statement are presented in Section 2 . A reminder is made of Curle's analogy as a diffraction problem and the proposed methodology for flow noise computation is detailed for general wave operators. Section 3 focuses on the numerical approximation of the Navier-Stokes and wave equations using FEM. The weak formulation of the equations is first exposed and then we proceed to their space and time discretization. In Section 4, two numerical examples are presented. The first one, used for verification and testing purposes, deals with aerodynamic noise generated by flow past a two-dimensional cylinder (aeolian tones). The second one concerns noise produced by flow past a sharp constraint at the exit of a three-dimensional rectangular duct. This second example is of importance to analyze the physics involved, for instance, in the generation of voice sibilant sounds like /s/. Conclusions close the paper in Section 5. 


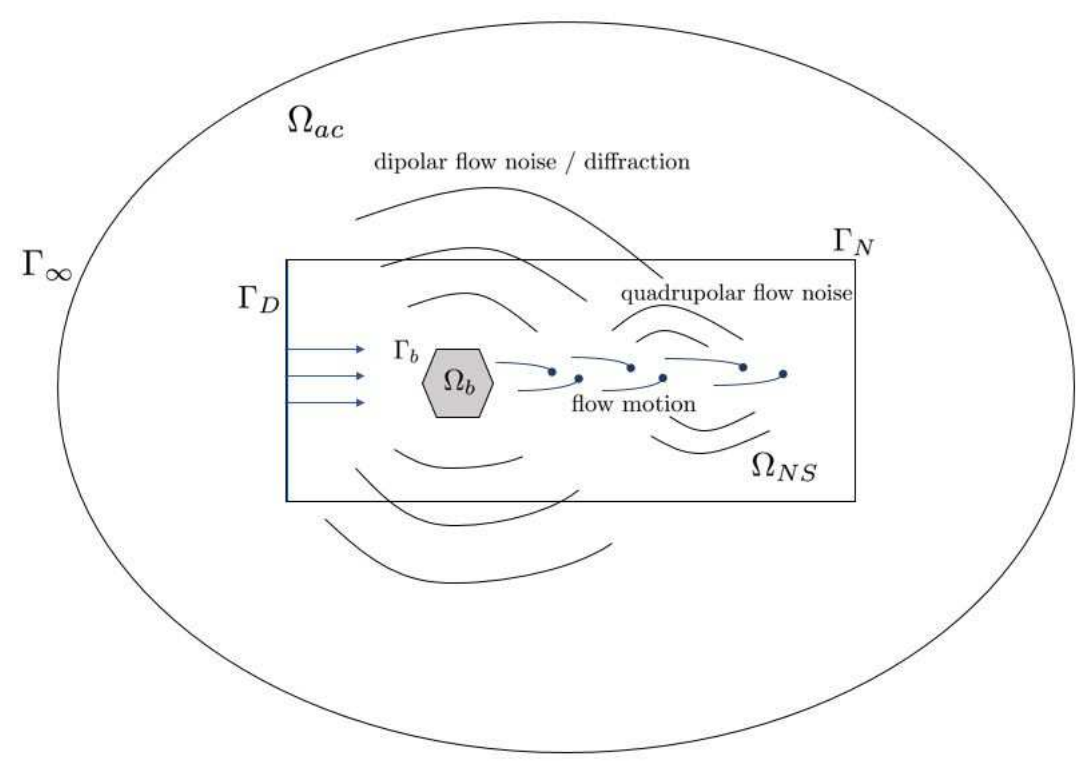

Figure 1: Flow noise problem. The dipolar contribution from the rigid body $\Omega_{b}$ in Curle's analogy corresponds to the diffraction of the quadrupolar flow noise generated at the wake past the body

\section{Problem statement}

\subsection{Curle's analogy as a diffraction problem}

As a motivation for this work, let us first review the connection between Curle's analogy and the diffraction of aerodynamic sound by a rigid body. We will closely follow [26] for that purpose. Consider the situation depicted in Fig. 1, where a low Mach, high Reynolds number gas flow impinges on a rigid body $\Omega_{b}$ and as a result, a wake develops past the body. The evolution of the aerodynamic velocity $\boldsymbol{u}^{0}(x, t)$ and the aerodynamic pressure $p^{0}(x, t)$ will be driven to a good extent by the incompressible Navier-Stokes equations. These are to be solved in a computational domain $\Omega_{N S} \subset \mathbb{R}^{d}$ (where $d=2,3$ is the number of space dimensions) with boundary $\partial \Omega_{N S}$ and prescribed initial and boundary conditions. Splitting $\partial \Omega_{N S}$ into three disjoint sets $\partial \Omega_{N S}=\Gamma_{b} \cup \Gamma_{D} \cup \Gamma_{N}$, the mathematical problem to be faced reads

$$
\begin{aligned}
\partial_{t} \boldsymbol{u}^{0}-\nu \Delta \boldsymbol{u}^{0}+\boldsymbol{u}^{0} \cdot \nabla \boldsymbol{u}^{0}+\nabla p^{0} & =\boldsymbol{f} & & \text { in } \Omega_{N S}, t>0, \\
\nabla \cdot \boldsymbol{u}^{0} & =0 & & \text { in } \Omega_{N S}, t>0, \\
\boldsymbol{u}^{0}(\boldsymbol{x}, 0) & =\boldsymbol{u}_{0}^{0}(\boldsymbol{x}) & & \text { in } \Omega_{N S}, t=0, \\
\boldsymbol{u}^{0}(\boldsymbol{x}, t) & =\mathbf{0} & & \text { on } \Gamma_{b}, t>0, \\
\boldsymbol{u}^{0}(\boldsymbol{x}, t) & =\boldsymbol{u}_{D}^{0}(\boldsymbol{x}, t) & & \text { on } \Gamma_{D}, t>0, \\
\boldsymbol{n} \cdot \boldsymbol{\sigma}(\boldsymbol{x}, t) & =\boldsymbol{t}_{N}(\boldsymbol{x}, t) & & \text { on } \Gamma_{N}, t>0,
\end{aligned}
$$

where $\nu$ stands for the kinematic viscosity and $\boldsymbol{f}$ for the external force. $\boldsymbol{\sigma}$ is the Cauchy stress tensor, and $\boldsymbol{n}$ and $\boldsymbol{t}_{N}$ respectively denote the outward normal at the boundary and the traction. $\partial_{t}$ denotes the first order time derivative.

In his celebrated work, Lighthill [8] reordered the compressible Navier-Stokes equations into a wave equation whose source term involved the double divergence of the so-called Lighthill's tensor, $T_{i j}$. In the particular case of low Mach and high Reynolds numbers one can neglect viscous dissipation and refraction in $T_{i j}$, and approximate the tensor solely in terms of the incompressible velocities

$$
T_{i j} \approx \rho_{0} u_{i}^{0} u_{j}^{0},
$$


with $\rho_{0}$ standing for the flow constant density. According to Lighthill's acoustic analogy we can then obtain the flow induced noise by solving the wave equation

$$
\begin{array}{r}
\partial_{t t}^{2} p-c_{0}^{2} \nabla^{2} p=\rho_{0} \partial_{i j}^{2}\left(u_{i}^{0} u_{j}^{0}\right) \quad \text { in } \Omega_{a c}, t>0, \\
\nabla p \cdot \boldsymbol{n}=0 \quad \text { on } \Gamma_{b}, t>0, \\
\nabla p \cdot \boldsymbol{n}=c_{0}^{-1} \partial_{t} p \quad \text { on } \Gamma_{\infty}, t>0, \\
p(\boldsymbol{x}, 0)=0, \partial_{t} p(\boldsymbol{x}, 0)=0 \quad \text { in } \Omega_{a c}, t=0 .
\end{array}
$$

In (3), $p$ represents the acoustic pressure and $c_{0}$ the speed of sound. $\Gamma_{\infty}$ corresponds to the outer boundary where a Sommerfeld like condition is to be imposed to avoid waves propagating outwards to reflect back into the computational domain. Besides, it is assumed for the time being that the source term in the r.h.s of (3a) vanishes outside $\Omega_{N S} \subset \Omega_{a c}$ (see section 2.3 for further considerations). The flow is therefore at rest in $\Omega_{a c} \backslash \Omega_{N S}$. On the other hand, we have used $\partial_{i}$ to denote the first order spatial derivative with respect to coordinate $x_{i}$, i.e., $\partial_{i} \equiv \partial / \partial x_{i}$. The summation convention for repeated indices is also assumed in what follows unless explicitly specified.

An integral solution to (3) could be found provided a tailored Green function $G(\boldsymbol{x}, t \mid \boldsymbol{y}, \tau)$ satisfying the boundary conditions at $\Gamma_{b}$ was known. This would result in the acoustic pressure field

$$
p(\boldsymbol{x}, t)=\rho_{0} \int_{-\infty}^{\infty} \int_{\Omega_{a c}} G(\boldsymbol{x}, t \mid \boldsymbol{y}, \tau) \partial_{i j}^{2}\left(u_{i}^{0} u_{j}^{0}\right) d^{3} \boldsymbol{y} d \tau
$$

Unfortunately, tailored Green functions are only available for bodies with very simple geometrical shapes. In the particular case of the typical wavelength of the generated sound being larger than the body principal dimension, one can make use of compact Green functions [28] and extend the number of situations where (4) could be applied in practice. Nonetheless, for more general applications it would be desirable to find a way out to solve (3) making use of the simpler free space Green function for the wave equation

$$
G_{F}(\boldsymbol{x}, t \mid \boldsymbol{y}, \tau)=\frac{1}{4 \pi|\boldsymbol{x}-\boldsymbol{y}|} \delta\left(t-\tau-\frac{|\boldsymbol{x}-\boldsymbol{y}|}{c_{0}}\right),
$$

where $\delta$ stands for the Dirac delta function. This was achieved by Curle [21] using an ingenious procedure, which allowed one to identify the rigid body contribution to flow noise with that of a dipole source distribution radiating into free space. Curle's final integral formulation for the acoustic pressure reads

$$
p(\boldsymbol{x}, t)=\rho_{0} \partial_{i j}^{2} \int_{\Omega_{a c}} \frac{1}{4 \pi|\boldsymbol{x}-\boldsymbol{y}|}\left[u_{i}^{0} u_{j}^{0}\right]_{t^{\prime}} d^{3} \boldsymbol{y}-\partial_{i} \int_{\Gamma_{b}} \frac{1}{4 \pi|\boldsymbol{x}-\boldsymbol{y}|}\left[p_{i j}^{\prime}\right]_{t^{\prime}} n_{j} d^{2} \boldsymbol{y},
$$

with $p_{i j}^{\prime}=P \delta_{i j}-\sigma_{i j}, P$ denoting the full compressible pressure to be evaluated at the body's boundary. As said, we are considering high Reynolds numbers so that $p_{i j}^{\prime} \approx P \delta_{i j}$ and the second term in the r.h.s of (6) simplifies to $\partial_{i} \int_{\Gamma_{b}}(4 \pi|\boldsymbol{x}-\boldsymbol{y}|)^{-1}[P]_{t^{\prime}} n_{i} d^{2} \boldsymbol{y}$. As usual for retarded potentials, the squared brackets in (6) denote evaluation at the retarded time $t^{\prime}:=t-|\boldsymbol{x}-\boldsymbol{y}| / c_{0}$.

According to (6), the acoustic pressure at a far field point in $\Omega_{a c} \backslash \Omega_{N S}$ has two contributions, namely the quadrupolar one directly stemming from flow motion (volume integral term in the r.h.s of (6)), and the dipolar one due to the presence of the rigid body $\Omega_{b}$ within the flow domain (surface integral term in the r.h.s). However, as commented in the Introduction, a severe difficulty appears for low Mach number flows if the acoustic source term is derived from an incompressible CFD computation. Whereas the incompressible velocity in the volume integral of (6) can be readily obtained from the solution of (1), this is not the case for the source term in the surface integral, because the integrand $P$ involves not only the aerodynamic pressure at $\Gamma_{b}$ but also the acoustic pressure fluctuations on it. In [23], an approximation was proposed to circumvent this problem that consists in making use of the incompressible pressure at the surface $\Gamma_{b}$ and in replacing the free field Green function for the wave equation (5) by that of the Poisson equation. An alternative to that approach will be proposed hereafter that involves less assumptions. This is based on the fact that, as noted by several authors (see e.g. $[24,25,26]$ ), the acoustic pressure contribution of the surface integral 
in (6) corresponds to the diffraction of the aerodynamic noise produced, in the case of Fig. 1, by the flow wake past the rigid body.

The tailored Green function in (4) can be decomposed as

$$
G(\boldsymbol{x}, t \mid \boldsymbol{y}, \tau)=G_{F}(\boldsymbol{x}, t \mid \boldsymbol{y}, \tau)+G_{D}(\boldsymbol{x}, t \mid \boldsymbol{y}, \tau),
$$

with $G_{F}$ being the free-space Green function in (5) and $G_{D}$ its diffracted (read also scattered) field correction that accounts for the presence of boundaries. Substituting (7) into (4) results in

$$
p(\boldsymbol{x}, t)=\rho_{0} \partial_{i j}^{2} \int_{\Omega_{a c}} \frac{1}{4 \pi|\boldsymbol{x}-\boldsymbol{y}|}\left[u_{i}^{0} u_{j}^{0}\right]_{t^{\prime}} d^{3} \boldsymbol{y}+\rho_{0} \int_{-\infty}^{\infty} \int_{\Omega_{a c}} G_{D} \partial_{i j}^{2}\left(u_{i}^{0} u_{j}^{0}\right) d^{3} \boldsymbol{y} d \tau .
$$

A direct comparison with (6) allows one to identify

$$
-\partial_{i} \int_{\Gamma_{b}} \frac{1}{4 \pi|\boldsymbol{x}-\boldsymbol{y}|}[P]_{t^{\prime}} n_{i} d^{2} \boldsymbol{y}=\rho_{0} \int_{-\infty}^{\infty} \int_{\Omega_{a c}} G_{D} \partial_{i j}^{2}\left(u_{i}^{0} u_{j}^{0}\right) d^{3} \boldsymbol{y} d \tau .
$$

It becomes then apparent that the Curle surface integral, which represents an acoustic dipole source distribution, does in fact correspond to the body diffraction of the turbulent noise generated by the flow wake.

\subsection{Proposed methodology}

In view of (8), one may consider the possibility of obtaining the individual flow quadrupolar turbulent contribution as well as the dipolar one due to diffraction all at once, as the outputs of a single FEM computational run. This would prove very useful in some aeroacoustics situations where the influence of each type of sources is needed, for example, for noise reduction remedial purposes, or for a better understanding of the underlying noise generation mechanisms of a given physical phenomenon.

Let us split the total acoustic pressure into its incident and diffracted components $p=p_{i}+p_{d}$ in $(3)$, as commonly done for diffraction problems. This results in the two wave equations,

$$
\begin{aligned}
\partial_{t t}^{2} p_{i}-c_{0}^{2} \nabla^{2} p_{i} & =\rho_{0} \partial_{i j}^{2}\left(u_{i}^{0} u_{j}^{0}\right) & & \text { in } \Omega_{a c} \cup \Omega_{b}, t>0, \\
\nabla p_{i} \cdot \boldsymbol{n} & =c_{0}^{-1} \partial_{t} p_{i} & & \text { on } \Gamma_{\infty}, t>0, \\
p_{i}(\boldsymbol{x}, 0) & =0, \partial_{t} p_{i}(\boldsymbol{x}, 0)=0 & & \text { in } \Omega_{a c} \cup \Omega_{b} t=0 .
\end{aligned}
$$

and

$$
\begin{aligned}
\partial_{t t}^{2} p_{d}-c_{0}^{2} \nabla^{2} p_{d} & =0 & & \text { in } \Omega_{a c}, t>0, \\
\nabla p_{d} \cdot \boldsymbol{n} & =-\nabla p_{i} \cdot \boldsymbol{n} & & \text { on } \Gamma_{b}, t>0, \\
\nabla p_{d} \cdot \boldsymbol{n} & =c_{0}^{-1} \partial_{t} p_{d} & & \text { on } \Gamma_{\infty}, t>0, \\
p_{d}(\boldsymbol{x}, 0)=0, \partial_{t} p_{d}(\boldsymbol{x}, 0) & =0 & & \text { in } \Omega_{a c}, t=0 .
\end{aligned}
$$

Because of the linearity of the problems, it is observed that $p_{d}+p_{i}$ is the solution of problem (3), restricting $p_{i}$ to domain $\Omega_{a c}$.

Instead of resorting to an integral formulation, it is herein proposed to solve the problem of aerodynamic sound generation by solving (1), (10) and (11) all together, in a single simulation. The weak form of these equations will be discretized in space using FEM and in time using finite difference schemes. At each time step of the simulation the incompressible velocity obtained from (1) will be used in (10) to compute the incident acoustic pressure field (i.e., the direct quadrupolar contribution), which in turn will be used in (11) to compute the diffracted pressure field (i.e., the direct dipolar surface contribution). Note that rigid boundaries have been assumed in the above formulation though acoustic impedance conditions can easily be taken into account, as it will be shown in the next subsection.

The depicted strategy avoids the problem of having to deal with the total pressure in the surface integral of Curle's analogy and independently provides the contributions $p_{i}$ and $p_{d}$ to the total acoustic pressure $p$, which could not have been obtained from the direct FEM solution of (3). Alternatively, one could also attempt at solving (3) and (10) and obtain the diffracted contribution by subtracting the incident acoustic pressure field from the total one. 


\subsection{Generalization to other wave operators and sound sources}

Though posing Curle's analogy as a diffraction problem has motivated the approach in the preceding section to compute the quadrupolar and dipolar contributions to aerodynamic sound, that methodology can be generalized to other linear wave operators and source terms that include phenomena neglected in Lighthill's tensor approximation (2). Let us denote by $L$ a generic linear wave operator acting on the acoustic pressure $p$ defined in $\Omega_{a c}, B_{b}$ a linear operator defining the boundary conditions at the rigid body (including e.g., a prescribed impedance value at $\Gamma_{b}$ ) and $B_{\infty}$ a linear operator describing a non-reflecting boundary condition at $\Gamma_{\infty}$. $Q$ will stand for a generic aerodynamic source term obtained from an incompressible CFD computation solving (1). Then, the aerodynamic noise problem of solving

$$
\begin{array}{r}
L p=Q \quad \text { in } \Omega_{a c}, t>0, \\
\nabla p \cdot \boldsymbol{n}=B_{b} p \quad \text { on } \Gamma_{b}, t>0, \\
\nabla p \cdot \boldsymbol{n}=B_{\infty} p \quad \text { on } \Gamma_{\infty}, t>0, \\
p(\boldsymbol{x}, 0)=0, \partial_{t} p(\boldsymbol{x}, 0)=0 \quad \text { in } \Omega_{a c}, t=0,
\end{array}
$$

can always be split using $p=p_{i}+p_{d}$ as

$$
\begin{array}{r}
L p_{i}=Q \quad \text { in } \Omega_{a c} \cup \Omega_{b}, t>0, \\
\nabla p_{i} \cdot \boldsymbol{n}=B_{\infty} p_{i} \quad \text { on } \Gamma_{\infty}, t>0, \\
p_{i}(\boldsymbol{x}, 0)=0, \partial_{t} p_{i}(\boldsymbol{x}, 0)=0 \quad \text { in } \Omega_{a c} \cup \Omega_{b} t=0,
\end{array}
$$

and

$$
\begin{array}{rc}
L p_{d}=0 & \text { in } \Omega_{a c}, t>0, \\
\nabla p_{d} \cdot \boldsymbol{n}-B_{b} p_{d}=-\nabla p_{i} \cdot \boldsymbol{n}+B_{b} p_{i} & \text { on } \Gamma_{b}, t>0, \\
\nabla p_{d} \cdot \boldsymbol{n}=B_{\infty} p_{d} & \text { on } \Gamma_{\infty}, t>0, \\
p_{d}(\boldsymbol{x}, 0)=0, \partial_{t} p_{d}(\boldsymbol{x}, 0)=0 \quad \text { in } \Omega_{a c}, t=0 .
\end{array}
$$

Equations (3), (10) and (11) are nothing but a particular case of (12), (13) and (14). Another example, could be that of identifying $L$ with the convective wave equation $L \equiv\left[c_{0}^{-2}\left(\partial_{t}+U_{0} \cdot \nabla\right)^{2}-\nabla^{2}\right]$ to account for the effects of a uniform mean flow velocity $U_{0}$ in the wave operator. With regard to the source term, one could consider, for instance, the double time derivative of the incompressible pressure instead of the double divergence of (2) as a source term, i.e., $Q \equiv c_{0}^{-1} \partial_{t t}^{2} p^{0}$ (see e.g., [29]). The boundary condition operator $B_{b}$ could involve the admittance coefficient at the surface, $\mu$, and be identified e.g., with $B_{b} \equiv-\mu c_{0}^{-1} \partial_{t}$. Besides, one could also attempt to extend the above procedure for the wave equation in mixed form [30], or for the more complex acoustic perturbation equations [17], though this is out of the scope of the present work.

Finally, we would like to note that in the case of dealing with $N$ diffracting disjoint bodies instead of just one, the problem becomes totally analogous to (13) and (14) if one is interested in knowing their quadrupolar and dipolar contributions as a whole. One simply has to consider that for such situations $\Omega_{b}=\bigcup_{j=1 \ldots N} \Omega_{b j}$ with $\bigcap_{j=1 \ldots N} \bar{\Omega}_{b j}=\emptyset$, each $\Omega_{b j}$ representing the $j$-th diffracting or scattering body (depending on the relation between wavelength and object characteristic size). An example of such a type would be that of determining the noise generated by a grille at the exit of a ventilation system, or knowing the influence of the upper and lower incisors in the voice generation of a fricative sound like /s/.

\section{Numerical discretization}

\subsection{Continuous weak formulation}

To present the numerical discretization of the proposed methodology we will focus on equations (1), (10) and (11). Resorting to a FEM approach for the spatial discretization of these equations demands working 
with their corresponding weak forms. Let us introduce some notation for that purpose. In what follows, for any two functions (or distributions) $f$ and $g$, the integral of their product over a domain $\omega$ will be designated by $(f, g)_{\omega} \equiv \int_{\omega} f g$. This integral will typically represent a scalar product or a duality pair. $H^{1}(\omega)$ will represent the space of functions whose distributional derivatives belong to $L^{2}(\omega)$. The vector counterparts of all these spaces will be indicated by bold letters. The regularity in time of time-dependent functions will not be explicitly displayed; it will be assumed that it is enough for the variational problems written below to make sense.

Let us consider the Navier-Stokes problem. Let $\mathcal{V}$ be space of functions in $\boldsymbol{H}^{1}\left(\Omega_{N S}\right)$ that satisfy the Dirichlet boundary conditions on $\Gamma_{b}$ and $\Gamma_{D}$, and $\mathcal{V}_{0}$ be space of functions in $\boldsymbol{H}^{1}\left(\Omega_{N S}\right)$ that vanish on these boundaries. The weak form of the Navier-Stokes equations (1) is found as usual by multiplying them by a test function $\boldsymbol{v}^{0}$ for the velocity and $q^{0}$ for the pressure, and integrating over the computational domain $\Omega_{N S}$. The variational problem to be solved is that of finding $\left[\boldsymbol{u}^{0}(\cdot, t), p^{0}(\cdot, t)\right] \in \mathcal{V} \times L^{2}\left(\Omega_{N S}\right)$ for all $t>0$ such that

$$
\begin{gathered}
\left(\partial_{t} \boldsymbol{u}^{0}, \boldsymbol{v}^{0}\right)_{\Omega_{N S}}+\left(\boldsymbol{u}^{0} \cdot \nabla \boldsymbol{u}^{0}, \boldsymbol{v}^{0}\right)_{\Omega_{N S}}+\nu\left(\nabla \boldsymbol{u}^{0}, \nabla \boldsymbol{v}^{0}\right)_{\Omega_{N S}}-\left(p^{0}, \nabla \cdot \boldsymbol{v}^{0}\right)_{\Omega_{N S}}=\left(\boldsymbol{f}, \boldsymbol{v}^{0}\right)_{\Omega_{N S}}+\left(\boldsymbol{t}_{N}, \boldsymbol{v}^{0}\right)_{\Gamma_{N}}, \\
\left(q^{0}, \nabla \cdot \boldsymbol{u}^{0}\right)_{\Omega_{N S}}=0,
\end{gathered}
$$

for all $\left[\boldsymbol{v}^{0}, q^{0}\right] \in \mathcal{V}_{0} \times L^{2}\left(\Omega_{N S}\right)$, and supplemented with the weak form of the initial conditions in (1c).

Analogously, multiplying (10) by the test function $q$ and integrating now over the domain $\Omega_{a c} \cup \Omega_{b}$ we get the variational problem of obtaining the incident acoustic pressure $p_{i}(\cdot, t) \in H^{1}\left(\Omega_{a c} \cup \Omega_{b}\right)$ for all $t>0$ which fulfills

$$
\left(\partial_{t t}^{2} p_{i}, q\right)_{\Omega_{a c} \cup \Omega_{b}}+c_{0}^{2}\left(\nabla p_{i}, \nabla q\right)_{\Omega_{a c} \cup \Omega_{b}}-c_{0}\left(\partial_{t} p_{i}, q\right)_{\Gamma_{\infty}}=\left(\boldsymbol{u}^{0} \cdot \nabla \boldsymbol{u}^{0}, \nabla q\right)_{\Omega_{a c} \cup \Omega_{b}},
$$

for all $q \in H^{1}\left(\Omega_{a c} \cup \Omega_{b}\right)$. Again, the weak forms of the initial conditions (10c) are to be considered to solve the problem.

Finally, the weak formulation for the diffracted acoustic pressure problem (11) becomes that of getting the pressure $p_{d}(\cdot, t) \in H^{1}\left(\Omega_{a c}\right)$ for all $t>0$ such that

$$
\left(\partial_{t t}^{2} p_{d}, q\right)_{\Omega_{a c}}+c_{0}^{2}\left(\nabla p_{d}, \nabla q\right)_{\Omega_{a c}}-c_{0}\left(\partial_{t} p_{d}, q\right)_{\Gamma_{\infty}}=c_{0}^{2}\left(\nabla p_{i} \cdot \boldsymbol{n}, q\right)_{\Gamma_{b}}
$$

for all $q \in H^{1}\left(\Omega_{a c}\right)$. In this case initial conditions (11d) have to be also appended to the variational form.

\subsection{Spatial discretization}

A FEM approach has been applied for the space discretization of all the involved variational equations in the preceding section. Even though different finite element meshes can be used for the three problems to be solved, we will write them all as $\{K\}, K$ standing for a generic element of diameter $h$. Summation for all the elements of the mesh will be written as $\sum_{K}$.

Let us first focus on the weak form of the Navier-Stokes equation in (15). For this case, it is wellknown that the standard Galerkin FEM solution suffers from many numerical problems. On the one hand, a compatibility inf-sup condition has to be satisfied to control the pressure that does not allow one to use equal interpolations for the incompressible velocity and pressure fields. On the other hand, instabilities also appear in the case of strong convection as happens for high Reynolds number flows. Numerical instabilities may also trigger at the early stage of evolutionary processes when using small time steps.

It is possible to solve the above difficulties by resorting to stabilized finite element approaches. In particular, the subgrid scale stabilization methods (also known as variational multiscale stabilization methods) $[31,32]$ are of special interest for the simulation of turbulent flows. This is so because, if well designed, they not only allow one to circumvent the above mentioned numerical problems, but also act as implicit large eddy simulation models $[32,33,34,35,36]$. The basic idea of subgrid scale methods is that of splitting the problem unknowns, $\boldsymbol{u}^{0}$ and $p^{0}$ for (15), and the test functions, $\boldsymbol{v}^{0}$ and $q^{0}$, into large scale components, $\boldsymbol{u}_{h}^{0}$ and $p_{h}^{0}$, which can be resolved by the computational mesh, and small scale components, $\tilde{\boldsymbol{u}}^{0}$ and $\tilde{p}^{0}$, which 
cannot be captured and whose effects onto the large scales have to be modeled. Substituting $\boldsymbol{u}^{0}=\boldsymbol{u}_{h}^{0}+\tilde{\boldsymbol{u}}^{0}$, $p^{0}=p_{h}^{0}+\tilde{p}^{0}, \boldsymbol{v}^{0}=\boldsymbol{v}_{h}^{0}+\tilde{\boldsymbol{v}}^{0}$ and $q^{0}=q_{h}^{0}+\tilde{q}^{0}$ in (15) yields two coupled equations, one driving the dynamics of the large scales but containing the influence of the small scales, and the other one governing the dynamics of the subscales, which in turn depend on the large scales. The solution to the latter is usually approximated considering the subscales to be directly proportional to the residual (in the algebraic subgrid scale (ASGS) version [31,37]) or taking the residual component perpendicular to the finite element space (in the orthogonal subgrid scale (OSS) approach [32]). The proportionality is characterized by means of a matrix of the so-called stabilization parameters.

For the simulations in the present work we have resorted to the ASGS formulation, assumed quasi-static subscales and neglected the influence of the pressure subscales. Let $\mathcal{V}_{h} \subset \mathcal{V}$ be the finite element space for the velocity, $\mathcal{V}_{h, 0} \subset \mathcal{V}_{0}$ for the velocity test functions and $\mathcal{Q}_{h} \subset L^{2}\left(\Omega_{N S}\right)$ for the pressure and pressure test functions. The space-discrete variational problem for the large scales then becomes that of finding $\left[\boldsymbol{u}_{h}^{0}(\cdot, t), p_{h}^{0}(\cdot, t)\right] \in \mathcal{V}_{h} \times \mathcal{Q}_{h}$ for all $t>0$ such that

$$
\begin{aligned}
& \left(\partial_{t} \boldsymbol{u}_{h}^{0}, \boldsymbol{v}_{h}^{0}\right)_{\Omega_{N S}}+\left(\boldsymbol{u}_{h}^{0} \cdot \nabla \boldsymbol{u}_{h}^{0}, \boldsymbol{v}_{h}^{0}\right)_{\Omega_{N S}}+\nu\left(\nabla \boldsymbol{u}_{h}^{0}, \nabla \boldsymbol{v}_{h}^{0}\right)_{\Omega_{N S}}-\left(p_{h}^{0}, \nabla \cdot \boldsymbol{v}_{h}^{0}\right)_{\Omega_{N S}}+\left(q_{h}^{0}, \nabla \cdot \boldsymbol{u}_{h}^{0}\right)_{\Omega_{N S}} \\
& \quad-\left(\boldsymbol{f}, \boldsymbol{v}_{h}^{0}\right)_{\Omega_{N S}}-\left(\boldsymbol{t}_{N}, \boldsymbol{v}_{h}^{0}\right)_{\Gamma_{N}} \\
& \quad+\left(\tilde{\boldsymbol{u}}^{0} \cdot \nabla \boldsymbol{u}_{h}^{0}, \boldsymbol{v}_{h}^{0}\right)_{\Omega_{N S}}-\left(\tilde{\boldsymbol{u}}^{0}, \tilde{\boldsymbol{u}}^{0} \cdot \nabla \boldsymbol{v}_{h}^{0}\right)_{\Omega_{N S}}-\sum_{K}\left(\tilde{\boldsymbol{u}}^{0}, \nu \Delta \boldsymbol{v}_{h}^{0}+\boldsymbol{u}_{h}^{0} \cdot \nabla \boldsymbol{v}_{h}^{0}+\nabla q_{h}^{0}\right)_{K}=0,
\end{aligned}
$$

for all $\left[\boldsymbol{v}_{h}^{0}, q_{h}^{0}\right] \in \mathcal{V}_{h, 0} \times \mathcal{Q}_{h}$. The incompressible velocity subscales $\tilde{\boldsymbol{u}}^{0}$ in (18) which solve the small scales equation are computed as

$$
\tilde{\boldsymbol{u}}^{0}=\tau\left(\boldsymbol{f}-\partial_{t} \boldsymbol{u}_{h}^{0}-\boldsymbol{u}_{h}^{0} \cdot \nabla \boldsymbol{u}_{h}^{0}+\nu \Delta \boldsymbol{u}_{h}^{0}-\nabla p_{h}^{0}\right),
$$

with the stabilization parameter $\tau$ being given by

$$
\tau=\left[c_{1} \frac{\nu}{h^{2}}+c_{2} \frac{\left|\boldsymbol{u}_{h}^{0}\right|}{h}\right]^{-1},
$$

and $c_{1}$ and $c_{2}$ in (15) standing for algorithmic parameters. From numerical experiments the values $c_{1}=4$ and $c_{2}=2$ have been deemed appropriate for them. The influence of the subscales $\tilde{\boldsymbol{u}}^{0}$ has been neglected in the non-linear term of the residual in (19), and in the stabilization parameter as well. Subscales are assumed to vanish at the interelement boundaries.

Observe that the first two lines of (18) are nothing but the Galerkin FEM approach to the variational Navier-Stokes problem, whereas the third line contains the subscale contributions to the material derivative, as well as the stabilization terms which mitigate the numerical problems mentioned above. The reader is referred to [35] for a detailed derivation of the above equations. These arise as a particular case of the most general situation in which subscales are tracked in time and all its non-linear contributions retained.

In what concerns the wave equations, (16) and (17) do not present special spatial discretization difficulties because they only involve the Laplacian operator. A standard Galerkin FEM proves accurate enough. Let $\mathcal{V}_{i h} \subset H^{1}\left(\Omega_{a c} \cup \Omega_{b}\right)$ and $\mathcal{V}_{d h} \subset H^{1}\left(\Omega_{a c}\right)$ be finite element spaces to approximate problems (16) and (17), respectively.

The spatial discrete version of (16) is that of finding $p_{i h}(\cdot, t) \in \mathcal{V}_{i h}$ for all $t>0$ which satisfies

$$
\left(\partial_{t t}^{2} p_{i h}, q_{h}\right)_{\Omega_{a c} \cup \Omega_{b}}+c_{0}^{2}\left(\nabla p_{i h}, \nabla q_{h}\right)_{\Omega_{a c} \cup \Omega_{b}}-c_{0}\left(\partial_{t} p_{i h}, q_{h}\right)_{\Gamma_{\infty}}=\left(\boldsymbol{u}_{h}^{0} \cdot \nabla \boldsymbol{u}_{h}^{0}, \nabla q_{h}\right)_{\Omega_{a c} \cup \Omega_{b}},
$$

for all $q_{h} \in \mathcal{V}_{i h}$. Analogously, the spatial discrete version of (17) consists in obtaining the diffracted pressure $p_{d h}(\cdot, t) \in \mathcal{V}_{d h}$ for all $t>0$ such that

$$
\left(\partial_{t t}^{2} p_{d h}, q_{h}\right)_{\Omega_{a c}}+c_{0}^{2}\left(\nabla p_{d h}, \nabla q_{h}\right)_{\Omega_{a c}}-c_{0}\left(\partial_{t} p_{d h}, q_{h}\right)_{\Gamma_{\infty}}=c_{0}^{2}\left(\nabla p_{i h} \cdot \boldsymbol{n}, q_{h}\right)_{\Gamma_{b}},
$$

for all $q_{h} \in \mathcal{V}_{d h}$ 
The main numerical difficulties with (21) and (22) usually arise from its time discretization (to be dealt with in the forthcoming subsection) or from undesired reflections at the boundary of the finite computational domain. Although simple Sommerfeld radiation conditions have proved precise enough for the numerical tests in the present study, the use of perfectly matched layers (PML) for the irreducible wave equation is certainly recommendable $[38,39,40]$.

Finally, we point out that to get the matrix versions of the above equations one has to expand as usual the unknowns $\boldsymbol{u}_{h}^{0}, p_{h}^{0}, p_{i h}, p_{d h}$, and their corresponding test functions, as linear combinations of polynomial basis functions and nodal unknowns, and rearrange (18), (21) and (22). Needless to say that the equations are to be supplied with the corresponding discrete initial conditions in weak form.

\subsection{Fully discretized numerical scheme in space and time}

To approximate the first and second order time derivatives in (18), (21) and (22) backward finite difference schemes of second order (BDF2) and of third order (BDF3) have been used. To fix notation, let us describe the former. Consider a partition of the time interval $[0, T]$ into $N$ equal time steps of size $\delta t:=t^{n+1}-t^{n}$ so that $0 \equiv t^{0}<t^{1}<\ldots<t^{n}<\ldots<t^{N} \equiv T$. Given a generic time dependent function $g(t)$, the following notation will be used for the BDF2 approximation to the first order time derivative:

$$
\left.\partial_{t} g\right|_{t^{n}} \approx \delta_{t} g^{n}:=\frac{1}{2 \delta t}\left(3 g^{n}-4 g^{n-1}+g^{n-2}\right),
$$

whilst the BDF2 second order time derivative will be approximated by

$$
\left.\partial_{t t}^{2} g\right|_{t^{n}} \approx \delta_{t t}^{2} g^{n}:=\frac{1}{\delta t^{2}}\left(2 g^{n}-5 g^{n-1}+4 g^{n-2}-g^{n-3}\right) .
$$

As usual, $g^{n}$ denotes an approximation of the evaluation of $g$ at time step $t^{n}$.

Given that the proposed concurrent FEM formulation for aeroacoustics is not intended for sound propagation at very far distances (for which an integral approach would probably be more efficient), the above BDF2 time discretization schemes prove accurate enough for the wave problems. Otherwise, if acoustic wave propagation was required for distances involving several tenths or hundreds of wavelengths, one should resort to high-order schemes (e.g., fourth order Runge-Kutta [41, 42]) or to symplectic integrators (e.g. of the Verlet-type).

At an arbitrary time step of the numerical simulation, the final fully discretized implicit scheme in space and time proposed to solve equations (3), (10) and (11) reads as follows. From known $\boldsymbol{u}_{h}^{0, n-2}$ and $\boldsymbol{u}_{h}^{0, n-1}$, compute the incompressible velocity and pressure at time step $t^{n},\left[\boldsymbol{u}_{h}^{0, n}, p_{h}^{0, n}\right] \in \mathcal{V}_{h} \times \mathcal{Q}_{h}$ such that

$$
\begin{aligned}
& \left(\delta_{t} \boldsymbol{u}_{h}^{0, n}, \boldsymbol{v}_{h}^{0}\right)_{\Omega_{N S}}+\left(\boldsymbol{u}_{h}^{0, n} \cdot \nabla \boldsymbol{u}_{h}^{0, n}, \boldsymbol{v}_{h}^{0}\right)_{\Omega_{N S}}+\nu\left(\nabla \boldsymbol{u}_{h}^{0, n}, \nabla \boldsymbol{v}_{h}^{0}\right)_{\Omega_{N S}}-\left(p_{h}^{0, n}, \nabla \cdot \boldsymbol{v}_{h}^{0}\right)_{\Omega_{N S}}+\left(q_{h}^{0}, \nabla \cdot \boldsymbol{u}_{h}^{0, n}\right)_{\Omega_{N S}} \\
& \quad-\left(\boldsymbol{f}^{n}, \boldsymbol{v}_{h}^{0}\right)_{\Omega_{N S}}+\left(\boldsymbol{t}_{N}^{n}, \boldsymbol{v}_{h}^{0}\right)_{\Gamma_{N}} \\
& \quad+\left(\tilde{\boldsymbol{u}}^{0, n} \cdot \nabla \boldsymbol{u}_{h}^{0, n}, \boldsymbol{v}_{h}^{0}\right)_{\Omega_{N S}}-\left(\tilde{\boldsymbol{u}}^{0, n}, \tilde{\boldsymbol{u}}^{0, n} \cdot \nabla \boldsymbol{v}_{h}^{0}\right)_{\Omega_{N S}}-\sum_{K}\left(\tilde{\boldsymbol{u}}^{0, n}, \nu \Delta \boldsymbol{v}_{h}^{0}+\boldsymbol{u}_{h}^{0, n} \cdot \nabla \boldsymbol{v}_{h}^{0}+\nabla q_{h}^{0}\right)_{K}=0,
\end{aligned}
$$

for all $\left[\boldsymbol{v}_{h}^{0, n}, q_{h}^{0, n}\right] \in \mathcal{V}_{h, 0} \times \mathcal{Q}_{h}$. Then, from known $\boldsymbol{u}_{h}^{0, n}, p_{i h}^{n-3}, p_{i h}^{n-2}$ and $p_{i h}^{n-1}$, find the incident acoustic pressure at time step $t^{n}, p_{i h}^{n} \in \mathcal{V}_{i h}$, that satisfies

$$
\left(\delta_{t t}^{2} p_{i h}^{n}, q_{h}\right)_{\Omega_{a c} \cup \Omega_{b}}+c_{0}^{2}\left(\nabla p_{i h}^{n}, \nabla q_{h}\right)_{\Omega_{a c} \cup \Omega_{b}}-c_{0}\left(\delta_{t} p_{i h}^{n}, q_{h}\right)_{\Gamma_{\infty}}=\left(\boldsymbol{u}_{h}^{0, n} \cdot \nabla \boldsymbol{u}_{h}^{0, n}, \nabla q_{h}\right)_{\Omega_{a c} \cup \Omega_{b}},
$$

for all $q_{h} \in \mathcal{V}_{i h}$. Finally, from $p_{i h}^{n}, p_{d h}^{n-3}, p_{d h}^{n-2}$ and $p_{d h}^{n-1}$, compute the diffracted acoustic pressure at time $t^{n}, p_{d h}^{n} \in \mathcal{V}_{d h}$, such that

$$
\left(\delta_{t t}^{2} p_{d h}^{n}, q_{h}\right)_{\Omega_{a c}}+c_{0}^{2}\left(\nabla p_{d h}^{n}, \nabla q_{h}\right)_{\Omega_{a c}}-c_{0}\left(\delta_{t} p_{d h}^{n}, q_{h}\right)_{\Gamma_{\infty}}=c_{0}^{2}\left(\nabla p_{i h}^{n} \cdot \boldsymbol{n}, q_{h}\right)_{\Gamma_{b}},
$$

for all $q_{h} \in \mathcal{V}_{d h}$.

Usually, knowing the acoustic field generated by the initial transients of the NS equations is of no interest at all, so the acoustic module in the above scheme (equations (26) and (27)) gets activated after a certain 


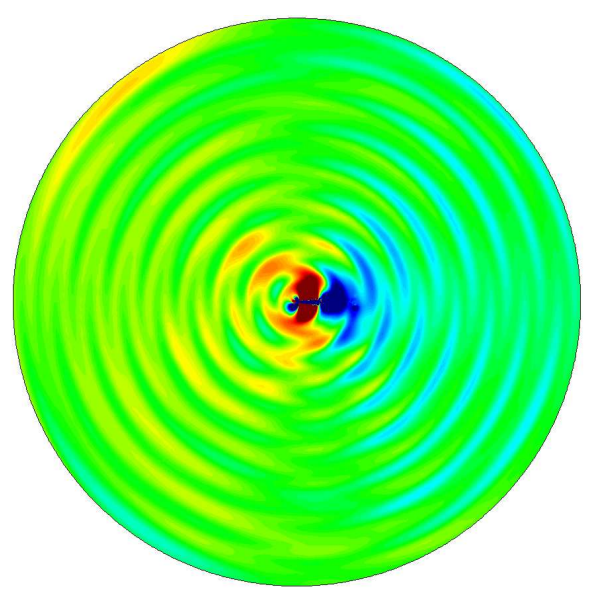

(a) Quadrupolar incident pressure

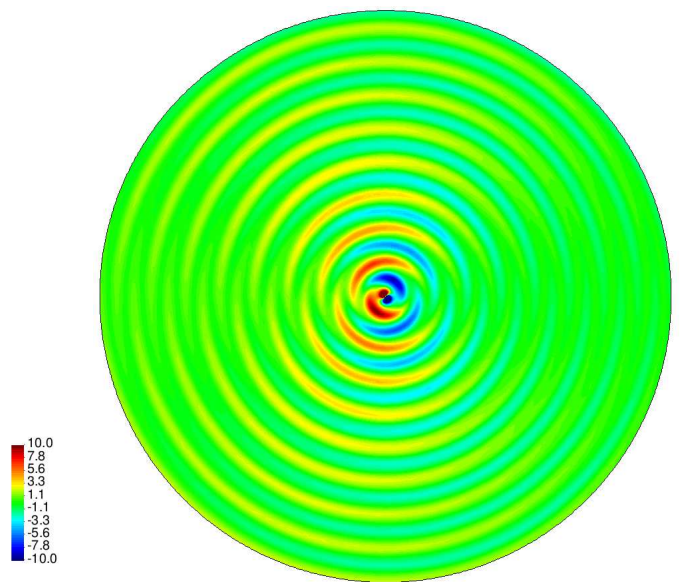

(b) Dipolar diffracted pressure
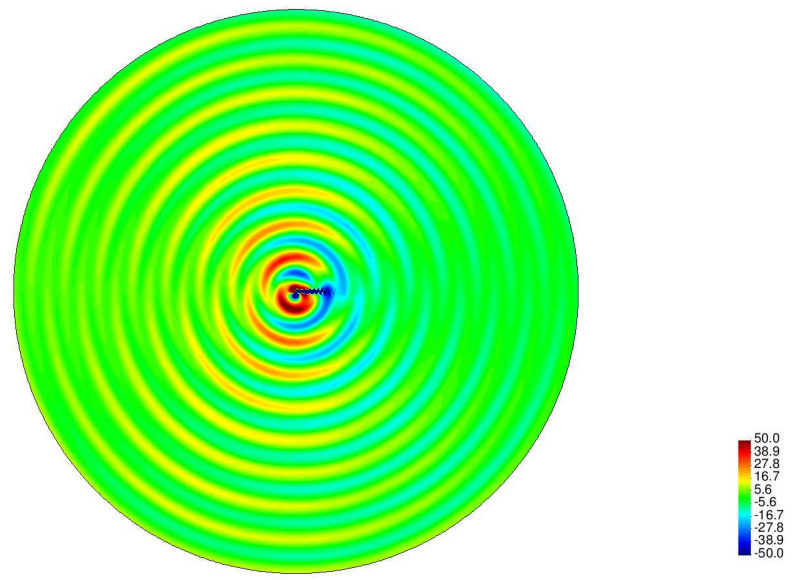

(c) Total acoustic pressure

Figure 2: Snapshots of the quadrupolar incident (a), dipolar diffracted (b) and total (c) acoustic pressure fields at time instant $t=0.3 \mathrm{~s}$. The total pressure in (c) is obtained from the summation of the incident and diffracted pressure fields in (a) and (b). The colour scale has not been kept constant for better visualization of the weak quadrupolar radiation.

period of time. On the other hand, note that at each time step of the simulation a linearization process is needed for the convective term in the NS equations. This can be achieved either by means of a simple Picard's scheme or by a Newton-Raphson's one.

Finally, it is worth mentioning that the proposed strategy presents a somehow tricky point because the computational domains for the variational NS equation (25) and for the two wave equations (26) and (27) are not the same. At each time step of the simulation, after solving (25) the domain $\Omega_{b}$ has to be switched on and included for the computation of the incident acoustic pressure in (26), and then switched off to compute the diffracted sound field in (27). Besides, it should be noted that special care should be taken when interpolating the results from the CFD domain to the acoustic one to avoid spurious errors [43, 44, 45].

\section{Numerical examples}

\subsection{Aeolian tones}

To test the above proposed methodology we will first address the classical case of aeolian tones induced by flow vortex shedding past a cylinder. The characteristic Reynolds number of the problem is $\operatorname{Re}=U^{0} D / \nu$, 


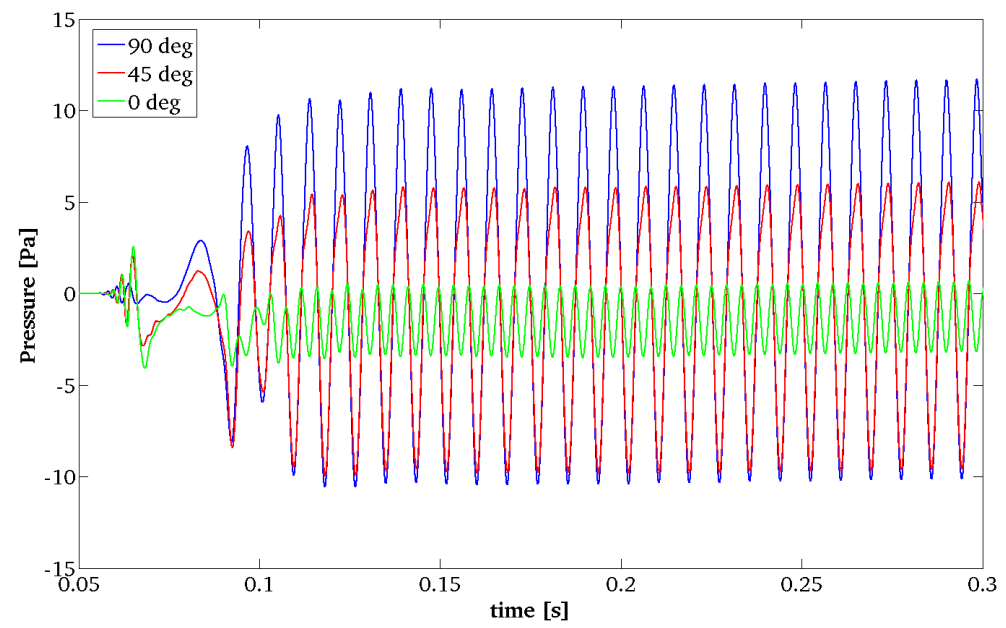

Figure 3: Evolution of the diffracted pressure at points $\left(20 \mathrm{~m}, 0^{\circ}\right)$, green line, $\left(20 \mathrm{~m}, 45^{\circ}\right)$, blue line and $\left(20 \mathrm{~m}, 90^{\circ}\right)$ red line.

with $U^{0}$ denoting the mean velocity impinging on the cylinder and $D$ its diameter. As long as Re is increased from an almost zero value, a set of bifurcations take place until the flow past the cylinder becomes fully turbulent at high enough Reynolds numbers (see e.g., [46]). Our interest is on that range of Re where the flow first loses its steadiness and a set of periodic vortices form behind the cylinder, known as the Von Kármán vortex street. The vortices are shed at an approximate frequency of $f_{s h}=S_{t} U^{0} / D$, with $S_{t}$ denoting the Strouhal number that slightly depends on Re as $S_{t}=0.198(1-19.7 / \operatorname{Re}$ ) (see e.g., [47]). The wake of vortices generate aerodynamic quadrupolar noise which is diffracted by the cylinder. The diffracted noise exhibits a strong dipolar character that clearly determines the acoustic pressure in the far field. The emitted sound has a dominant frequency of $f_{s h}$. Aeolian tones can be appreciated in practice when wind impinges power transmission lines and are also emitted, for instance, by train pantographs and tubular heat exchangers.

For the simulations we have considered a two-dimensional cylinder with diameter $D=0.1 \mathrm{~m}$ embedded in a circular acoustic computational domain $\Omega_{a c}$ that has a diameter of $D_{\Omega_{a c}}=50 \mathrm{~m}$. An inner rectangular domain $\Omega_{N S}$ of dimensions $5 \mathrm{~m} \times 10.4 \mathrm{~m}$ has been used for the CFD computation. An inlet flow velocity of $U^{0}=50 \mathrm{~m} / \mathrm{s}$ has been prescribed in the horizontal direction on its Dirichlet boundary, which has resulted in a vortex shedding and emitted sound frequency of $120 \mathrm{~Hz}$. We have considered a sound speed of $c_{0}=343 \mathrm{~m} / \mathrm{s}$. The two-dimensional mesh for $\Omega_{N S}$ consists of 55881 linear finite elements while 194322 linear elements have been used for the acoustic domain $\Omega_{a c}$. A time step of $\delta t=10^{-4} \mathrm{~s}$ has been chosen for the time evolution. The variational equations (15), (16) and (17) have been solved following the explanations in Sections 3.2 and 3.3.

Representative snapshots at time instant $t=0.3 \mathrm{~s}$ of the generated acoustic pressure field have been plotted in Fig. 2. In Fig. 2a we present the incident acoustic pressure field generated by the vortex wake which exhibits a clear quadrupolar directivity pattern. We remind that this computation is done in $\Omega_{a c} \cup \Omega_{b}$ as if the cylinder was absent. The incident acoustic pressure is diffracted by the cylinder which emits aerodynamic sound with a dipole pattern. The colour scale in Fig. 2a has been set different from that in Fig. 2b to better observe the quadrupolar radiation. It is to be noted that the amplitude of the diffracted sound turns to be $\sim 6-7$ times stronger than the incident one, which is in accordance with the fact that the dipolar contribution is of order $M^{-1}$ times the quadrupolar one (see e.g., [26]). In the present example the Mach number is $M \approx 0.146$.

The total acoustic field has been plotted in Fig. 2c. As observed, except for the area just past the cylinder, the results in Fig. 2c almost totally match those in Fig. 2b confirming that the far field is governed by the dipolar diffracted noise. This can also be appreciated in Fig. 3, where we have plotted the acoustic pressure evolution at three points located at distances and angles with respect to the horizontal of $\left(20 \mathrm{~m}, 0^{\circ}\right)$, $\left(20 \mathrm{~m}, 45^{\circ}\right)$ and $\left(20 \mathrm{~m}, 90^{\circ}\right)$ from the center of the cylinder. The acoustic pressure takes its maximum 


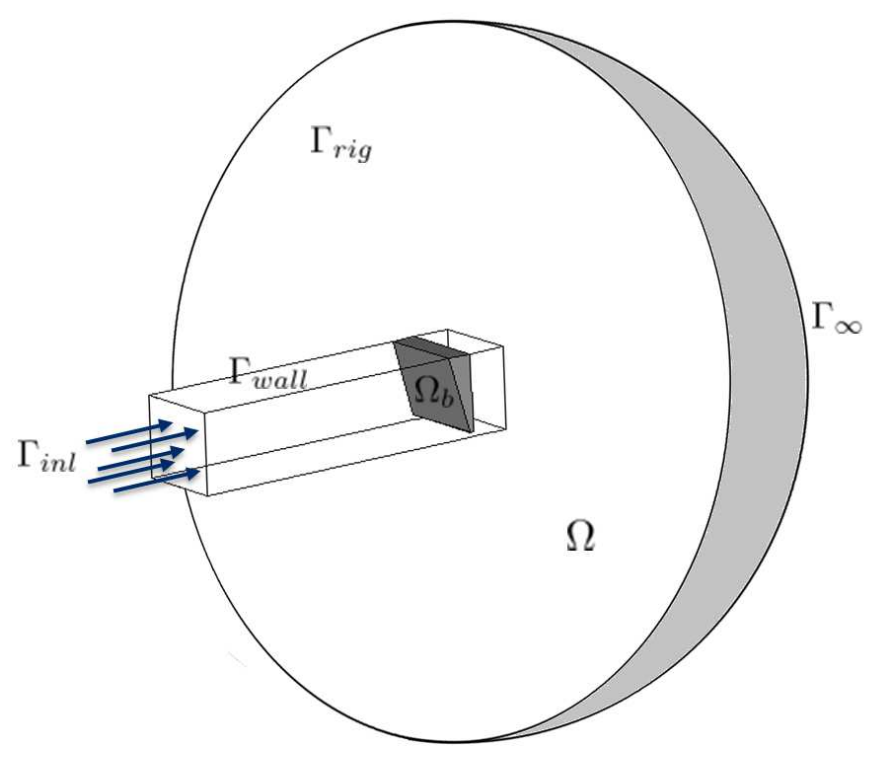

Figure 4: Sketch of the computational domain for the 3D duct with sharp constraint

amplitude at the axis perpendicular to the inlet inflow direction.

\subsection{D duct exit with sharp constraint}

The second application case focuses on the validation of the presented method for a benchmark test employed for better understanding the aeroacoustics in the generation of the sibilant sound /s/. The test consists of a duct with a sharp edge obstacle stuck at the end, which may be roughly viewed as a very idealized representation of a vocal tract and the teeth [48]. When the flow passes through the gap left by the constriction a turbulent wake develops driven by a shedding frequency. This results in the generation of aerodynamic noise which becomes diffracted by the teeth. As it will be shown, it will be again this diffracted noise component the one that dominates the far field acoustics.

The computational domain for both, the CFD and acoustic computations, consists of a three dimensional rectangular duct with dimensions $10.2 \mathrm{~cm} \times 2.5 \mathrm{~cm} \times 2.5 \mathrm{~cm}$ and a hemisphere at its exit, $15 \mathrm{~cm}$ in radius,

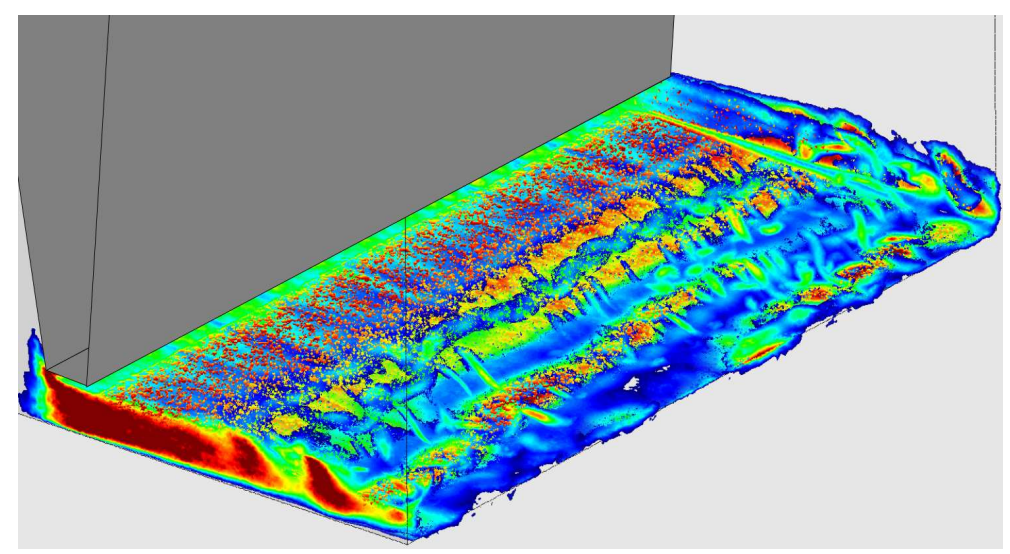

Figure 5: 3D isovorticity surfaces 


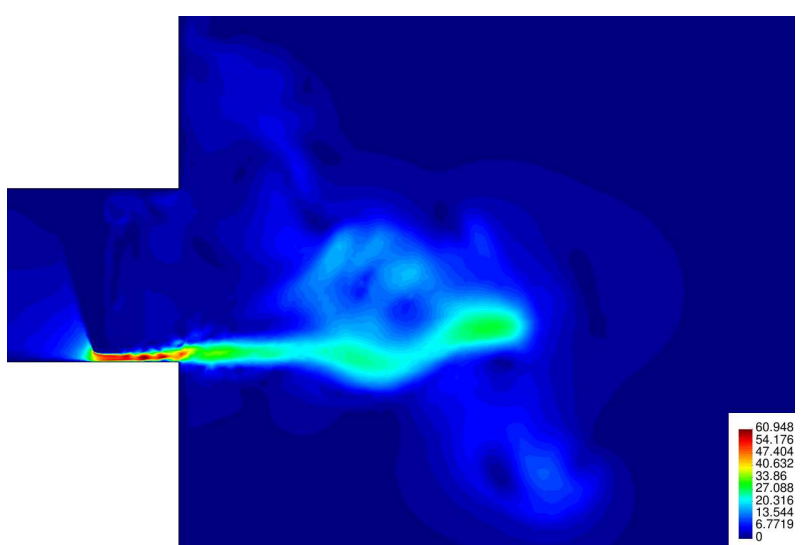

(a)

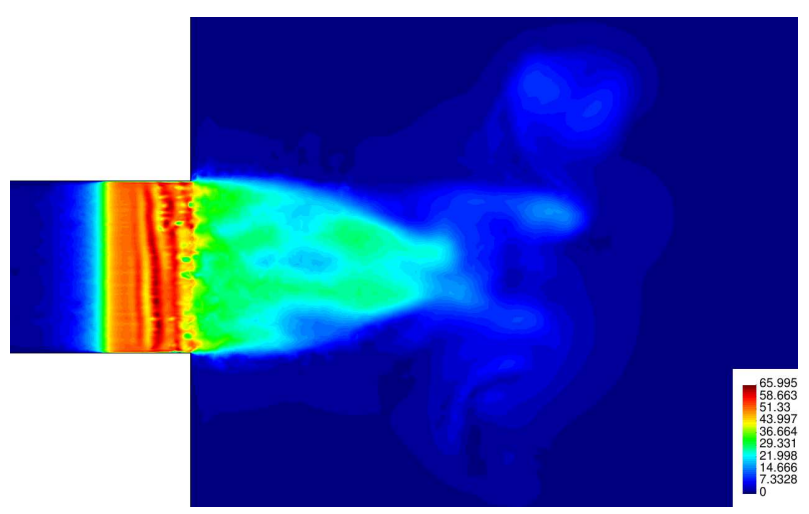

(c)

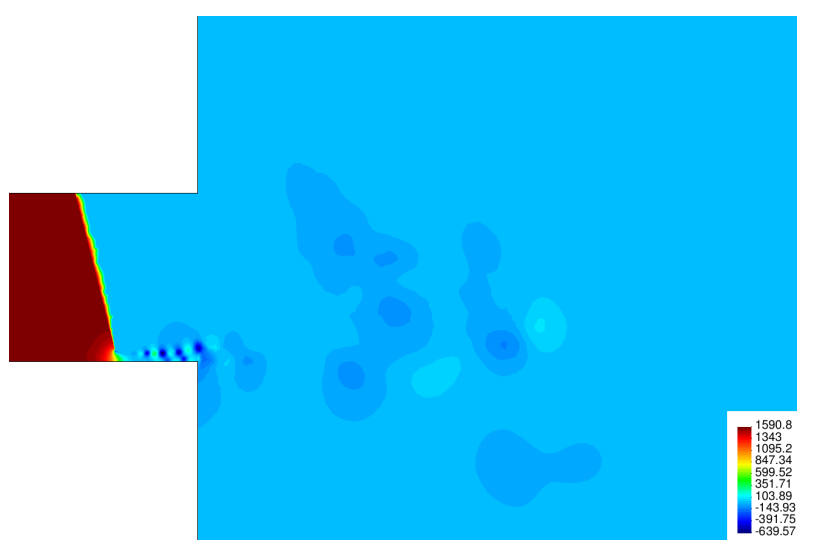

(b)

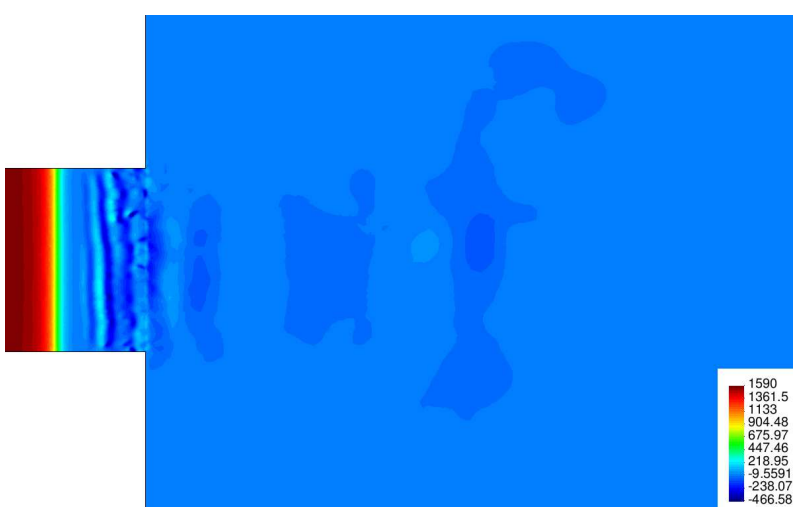

(d)

Figure 6: Velocity profile at $z=0.0125 \mathrm{~m}$ (a), velocity profile at $y=0.0032 \mathrm{~m} \mathrm{(b),} \mathrm{pressure} \mathrm{profile} \mathrm{at} z=0.0125 \mathrm{~m}$ (c) and pressure profile at $y=0.0032 \mathrm{~m}(\mathrm{~d})$ at $t=0.006 \mathrm{~s}$.

to account for outward acoustic wave propagation. The teeth is placed close to the duct exit leaving a gap of $0.15 \mathrm{~cm} \times 2.5 \mathrm{~cm}$ which corresponds to $6 \%$ of the duct section (see Fig. 4). The thickness of the edge of the teeth is $1.25 \mathrm{~mm}$. As regards the CFD computation, an inlet velocity of $(2.4,0,0)^{\top} \mathrm{m} / \mathrm{s}$ is imposed for the air at the duct entrance whereas a no-slip boundary condition is prescribed on its walls. The Reynolds number according to the height of the teeth gap and the inlet velocity is $R e=300$. In what concerns the acoustic simulations, the duct walls have been assumed to be rigid and a Sommerfeld boundary condition has been imposed at the outer surface of the hemisphere. The computational mesh is made of 42550677 tetrahedral linear finite elements with 6798782 nodes, using equal interpolation for velocity and pressure. A second order fractional step scheme has been used to solve the incompressible Navier Stokes equations, using a biconjugate gradient solver with a Hypre Pilut preconditioner for the velocity and a Trilinos ML one for the pressure. The later has been also used for the acoustic computations. In what refers the time discretization, a BDF3 scheme has been used for the CFD simulations and a BDF2 for the wave equation. The time step is given by $\delta t=2 \times 10^{-6} \mathrm{~s}$. The CFD has been first run for 1000 steps to surpass the initial transients without triggering the acoustic module. Both, CFD and acoustic results have been then computed from the subsequent 2000 time steps. All simulations have been performed using 1024 processors of a PDC Beskow machine at the KTH (Kungliga Tekniska Högskolan) supercomputing center.

As a result of the CFD computation, and as mentioned above, a turbulent wake develops past the teeth with some dominating shedding frequencies, the most important one taking place at $6 \mathrm{kHz}$. In Fig. 5 we show a snapshot of the isovorticity surfaces of the flow just past the teeth where the formation of coherent vortex structures can be appreciated. In Figs. 6a and c, we respectively present a vertical cut (at $z=0.0125 \mathrm{~m}$ ) and 


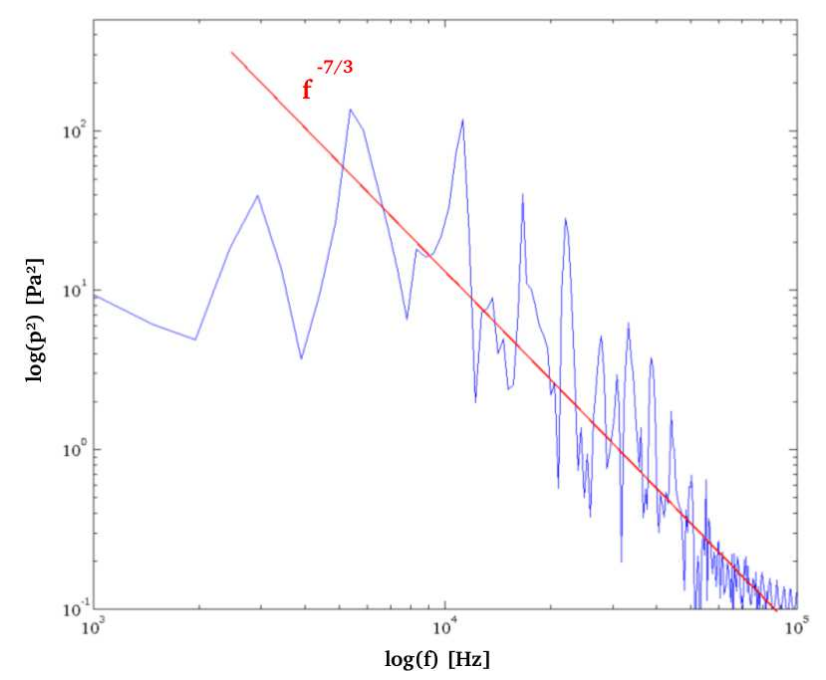

Figure 7: Point pressure spectrum $E_{p p}$ in $\left[\mathrm{Pa}^{2}\right]$ versus frequency $f$ in $\mathrm{Hz}$.

a horizontal cut (at $y=0.0032 \mathrm{~m}$ ) for the velocity modulus, whereas the analogous ones for the aerodynamic pressure are given in Figs. $6 \mathrm{~b}$ and $\mathrm{d}$. All of them are plotted at $t=0.006 \mathrm{~s}$. In Fig. 7 we show the pressure spectrum $E_{p p}$ in a log-log plot for a point located at the flow wake. According to Kolmogorov's theory, $E_{p p} \sim k^{-7 / 3}, k$ being the wavenumber, and making use of the frozen-turbulence approximation (Taylor's hypothesis) it can be shown that the dependency with frequency also becomes $E_{p p} \sim f^{-7 / 3}$ (see e.g., [49, 50]). The slope $-7 / 3$ is plotted as a red line in Fig. 7. As it can be observed, the computed spectrum closely matches the Kolmogorov pressure spectrum prediction for fully developed isotropic turbulence.

The generated aerodynamic sound results in acoustic waves propagating outside the duct exhibiting a

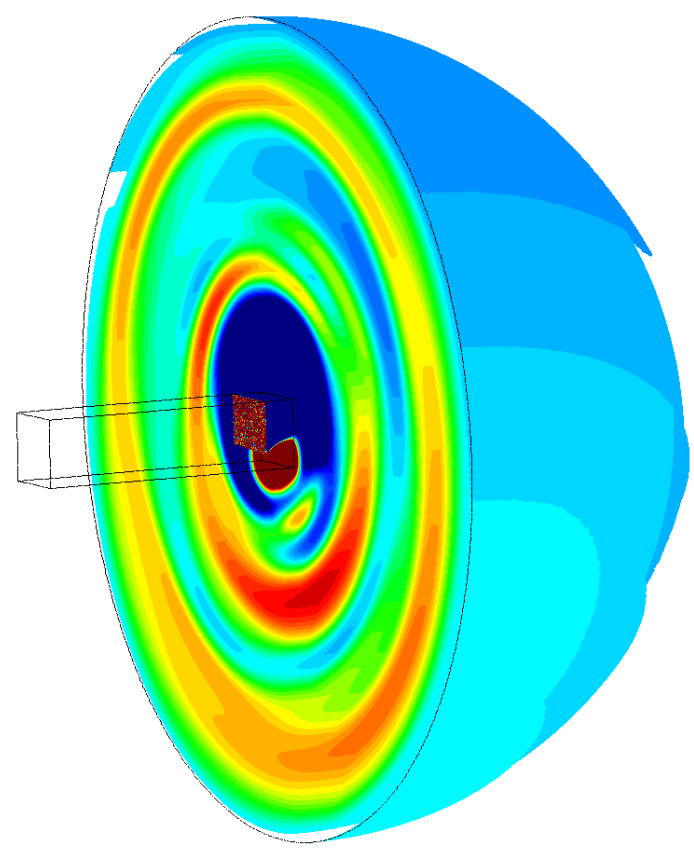

Figure 8: Total acoustic pressure front waves 


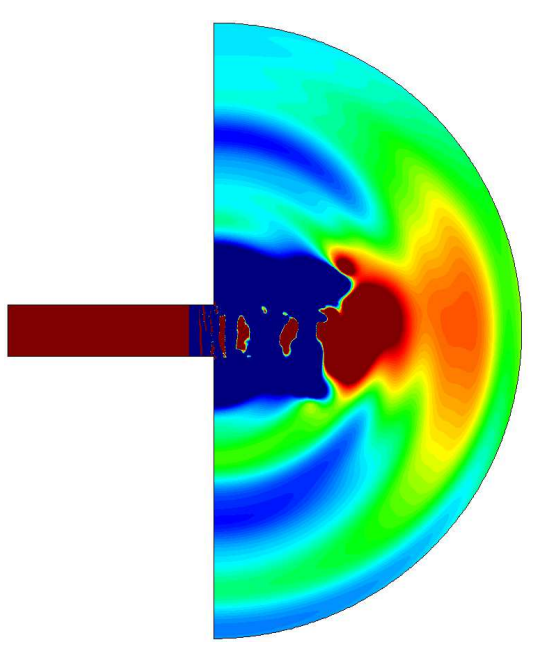

(a)

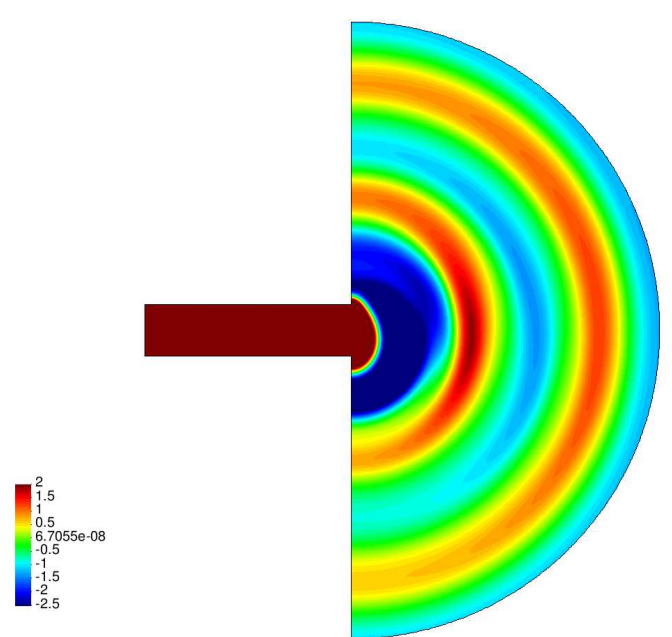

(b)

Figure 9: Incident acoustic pressure at $y=0.0032 \mathrm{~m}$ (a) and diffracted acoustic pressure at $y=0.0032 \mathrm{~m}(\mathrm{~b})$ for $\mathrm{t}=0.006 \mathrm{~s}$.

spherical directivity pattern (see Fig. 8). In Fig. 9a and b we respectively plot the incident and diffracted acoustic pressure for the plane located at $y=0.0032 \mathrm{~m}$. The influence of the wake is apparent for the incident field whereas it is logically absent for the diffracted acoustic field. Again, we should remind that the incident acoustic field computation is carried out by removing the teeth from the computational domain. It is to be noted that Lighthill's source term becomes strongly concentrated at the wake past the teeth and quickly smears out when the flow leaves the duct (see Fig. 10).

One can easily check that it is the noise diffracted by the teeth the one that governs the acoustics at the far field, in accordance with theoretical models for sibilant sound production (see e.g., [51]). In Fig. 11a we have plotted a sample of the total, incident and diffracted time history for the acoustic pressure at a point located at the far field. As observed, the diffracted component almost justifies the total acoustic pressure at this point. In Fig. 11b we present the Fourier transform for the incident and diffracted acoustic pressure evolution. The diffracted spectrum presents a clear peak at $6 \mathrm{kHz}$, corresponding to the dominant shedding frequency in the turbulent wake (see Fig. 7). This frequency determines the main oscillations observed in the diffracted and total pressure evolution in Fig. 11a.

Finally, it should be remarked that despite of the present example being able to reproduce the basic

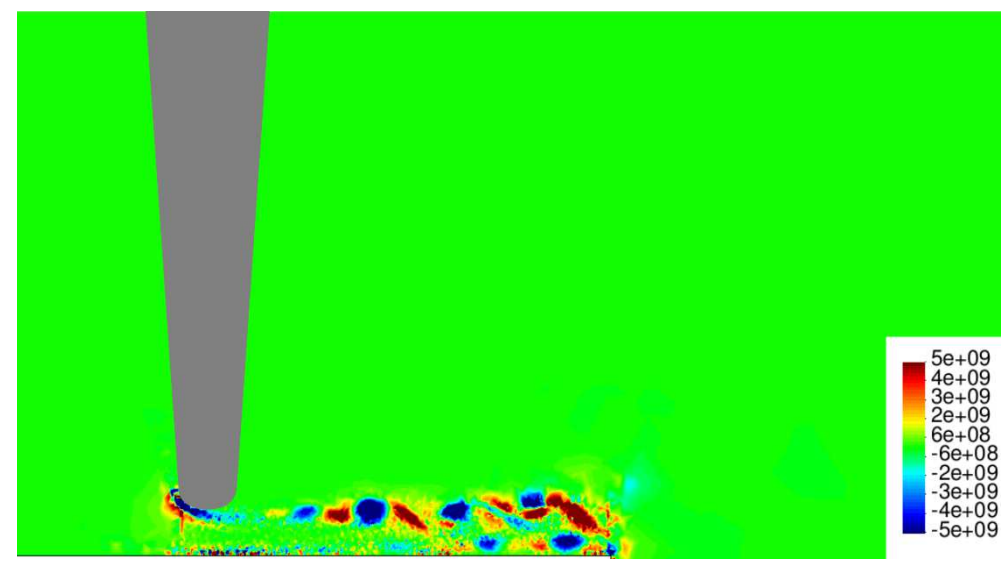

Figure 10: Lighthill source term $\rho_{0} \partial_{i j}^{2}\left(u_{i}^{0} u_{j}^{0}\right)$ at plane $z=0.0125 \mathrm{~m}$. 


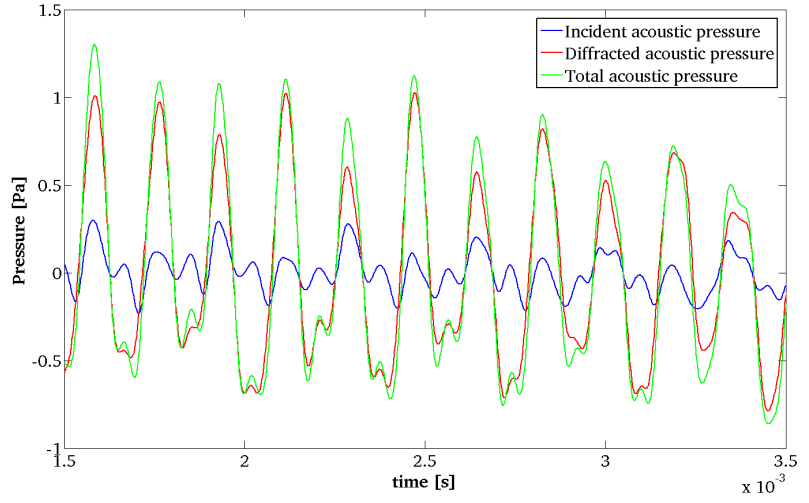

(a)

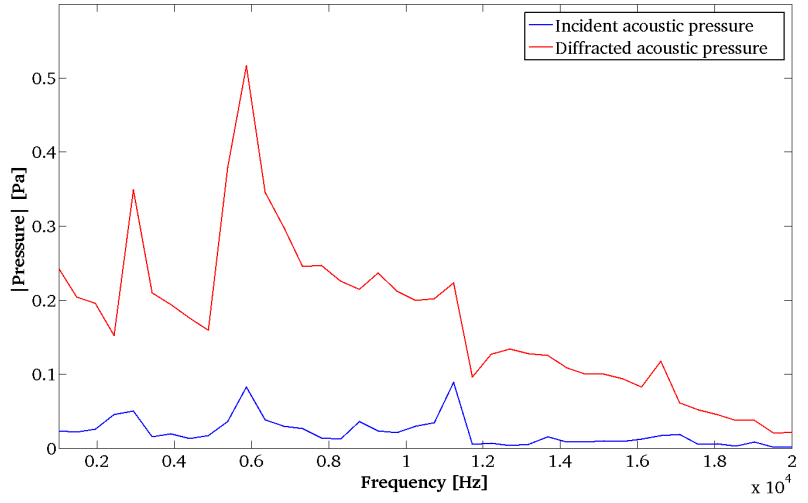

(b)

Figure 11: Time history for the total, incident and diffracted acoustic pressure at a far field point (a) and Fourier transform of the incident and diffracted acoustic components (b).

mechanism of sibilant sound generation, the obtained spectrum differs from those of realistic experimental data, obtained from human being recordings. For instance, in [52] several frequency spectra of phoneme /s/ were recorded for different different languages, syllables, ages and gender. The spectra in that study were rather flat within the range of $2-10 \mathrm{kHz}$, peaking slighthly between $6.8-8 \mathrm{kHz}$. It is expected that when running the current simulations using realistic human vocal tract geometries for sibilant $/ \mathrm{s} /$ the obtained spectra will be closer to the measured ones. Yet this is out of the scope of the current work and it is left for future developments.

\section{Conclusions}

This paper suggests a methodology to obtain the turbulent quadrupolar contribution to aerodynamic flow noise, as well as the dipolar one due to the influence of rigid body surfaces, as a direct output of a single finite element computational run. To that purpose use is made of Lighthill's acoustic analogy, although as shown, the procedure can be extended to any other acoustic linear wave operator and acoustic source term. Instead of directly solving the wave equation for the acoustic pressure, the latter becomes split into an incident component, due to flow motion, plus a diffracted one, due to the presence of bodies within the flow. At each time step of the simulation, the incompressible Navier-Stokes equations are solved and an approximation to Lighthill's tensor is derived from them. This source term is inserted in a wave equation for the incident pressure that is solved as if the rigid body was absent for that time step. This provides the quadrupolar flow noise contribution. The value of the incident pressure at the boundary of the rigid body is then used to compute the dipolar acoustic pressure contribution due to the body's diffraction.

The proposed approach avoids the problem of the standard Curle formulation for low Mach number aeroacoustics, of knowing the acoustic pressure fluctuations in the surface integral, not just the incompressible pressure that could stem from an incompressible CFD simulation.

\section{Acknowledgments}

This work is supported by EU-FET grant EUNISON 308874. The second author would like also to acknowledge the Generalitat de Catalunya (SUR/ECO) for the predoctoral FI Grant no. 2015 FI-B 00227, and the fourth author acknowledges the support received from the ICREA Acadèmia Program, from the Catalan Government. The authors thankfully acknowledge the computer resources, technical expertise and assistance provided by the Red Española de Supercomputación (RES-BSC) as well as the KTH (Kungliga Tekniska Högskolan) supercomputing center. 


\section{References}

[1] C. Bogey, C. Bailly, D. Juvé, Numerical simulation of sound generated by vortex pairing in a mixing layer, AIAA J.38 (12) (2000) 2210-2218.

[2] C. Bailly, C. Bogey, X. Gloerfelt, Some useful hybrid approaches for predicting aerodynamic noise, C. R. Mec.333 (9) (2005) 666-675.

[3] A. A. Oberai, F. Roknaldin, T. J. R. Hughes, Computation of trailing-edge noise due to turbulent flow over an airfoil, AIAA J.40 (11) (2002) 2206-2216.

[4] O. Guasch, R. Codina, An algebraic subgrid scale finite element method for the convected Helmholtz equation in two dimensions with applications in aeroacoustics, Comput. Methods Appl. Mech. Engrg.196(45-48) (2007) 4672-4689.

[5] O. Guasch, R. Codina, Computational aeroacoustics of viscous low speed flows using subgrid scale finite element methods, J. Comput. Acoust.17(3) (2009) 309-330.

[6] M. Kaltenbacher, M. Escobar, S. Becker, I. Ali, Numerical simulation of flow-induced noise using LES/SAS and lighthill's acoustic analogy, Int. J. Numer. Meth. Fluids63 (9) (2010) 1103-1122.

[7] J. Baiges, R. Codina, A variational multiscale method with subscales on the element boundaries for the Helmholtz equation, Int. J. Numer. Meth. Engrg.93 (6) (2013) 664-684.

[8] M. J. Lighthill, On sound generated aerodynamically. I. general theory, Proc. R. Soc. Lond. A211 (1107) (1952) $564-587$.

[9] S. Crow, Aerodynamic sound emission as a singular perturbation problem, Stud. Appl. Math.49 (1) (1970) $21-44$.

[10] J. Ristorcelli, A closure for the compressibility of the source terms in Lighthill's acoustic analogy, Tech. Rep. ICASE Report 97-44, NASA/CR-1997-201738 (1997).

[11] S. A. Slimon, M. C. Soteriou, D. W. Davis, Development of computational aeroacoustics equations for subsonic flows using a mach number expansion approach, J. Comput. Phys.159 (2) (2000) 377-406.

[12] A. Powell, Theory of vortex sound, J. Acoust. Soc. Am.36 (1) (1964) 177-195.

[13] M. Howe, The generation of sound by aerodynamic sources in an inhomogeneous steady flow, J. Fluid Mech.67 (03) (1975) $597-610$.

[14] W. Möhring, On vortex sound at low Mach number, J. Fluid Mech.85 (04) (1978) 685-691.

[15] G. Lilley, On the noise radiated from a turbulent high speed jet, in Computational Aeroacoustics, J.C. Hardin and M.Y. Hussaini (Eds.), Springer Verlag, 1993.

[16] M. Goldstein, A generalized acoustic analogy, J. Fluid Mech.488 (2003) 315-333.

[17] R. Ewert, W. Schröder, Acoustic perturbation equations based on flow decomposition via source filtering, J. Comput. Phys.188 (2) (2003) 365-398.

[18] A. Hueppe, M. Kaltenbacher, Spectral finite elements for computational aeroacoustics using acoustic perturbation equations, J. Comput. Acoust.20 (02) (2012) 1240005.

[19] O. Guasch, P. Sánchez-Martín, A. Pont, J. Baiges, R. Codina, Residual-based stabilization of the finite element approximation to the acoustic perturbation equations for low Mach number aeroacoustics, Submitted.

[20] C. Bailly, C. Bogey, Contributions of computational aeroacoustics to jet noise research and prediction, Int. J. Comput. Fluid Dyn.18 (6) (2004) 481-491.

[21] N. Curle, The influence of solid boundaries upon aerodynamic sound, Proc. R. Soc. Lond. A231 (1187) (1955) 505-514.

[22] J. F. Williams, D. L. Hawkings, Sound generation by turbulence and surfaces in arbitrary motion, Phil. Trans. Roy. Soc. A264 (1151) (1969) 321-342.

[23] P. Martínez-Lera, C. Schram, H. Bériot, R. Hallez, An approach to aerodynamic sound prediction based on incompressibleflow pressure, J. Sound Vib.333 (1) (2014) 132-143.

[24] P. Doak, Acoustic radiation from a turbulent fluid containing foreign bodies, Proc. R. Soc. Lond. A254 (1276) (1960) $129-146$.

[25] D. Crighton, Basic principles of aerodynamic noise generation, Prog. Aerosp. Sci.16 (1) (1975) 31-96.

[26] X. Gloerfelt, F. Pérot, C. Bailly, D. Juvé, Flow-induced cylinder noise formulated as a diffraction problem for low Mach numbers, J. Sound Vib.287 (1) (2005) 129-151.

[27] O. Guasch, A. Pont, J. Baiges, R. Codina, Concurrent finite element simulation of incident and diffracted flow noise in computational aeroacoustics, in: InterNoise2015, San Francisco, California, USA, 2015.

[28] M. S. Howe, Theory of vortex sound, Vol. 33, Cambridge University Press, 2003.

[29] M. Roger, Aeroacoustics: Some theoretical background-The acoustic analogy, J. Anthoine and T. Colonius (Eds.), Vol. LS 2006-05, Von Karman Institute, Rhode-St-Genese, 2006.

[30] R. Codina, Finite element approximation of the hyperbolic wave equation in mixed form, Comput. Methods Appl. Mech. Engrg.197(13-16) (2008) 1305-1322.

[31] T. Hughes, Multiscale phenomena: Green's function, the dirichlet-to-neumann formulation, subgrid scale models, bubbles and the origins of stabilized formulations, Comput. Methods Appl. Mech. Engrg.127 (1995) 387-401.

[32] R. Codina, Stabilized finite element approximation of transient incompressible flows using orthogonal subscales, Comput. Methods Appl. Mech. Engrg.191 (2002) 4295-4321.

[33] J. Hoffman, C. Johnson, A new approach to computational turbulence modeling, Comput. Methods Appl. Mech. Engrg.195 (23) (2006) 2865-2880.

[34] Y. Bazilevs, V. Calo, J. Cottrell, T. Hughes, A. Reali, G. Scovazzi, Variational multiscale residual-based turbulence modeling for large eddy simulation, Comput. Methods Appl. Mech. Engrg.197(1-4) (2007) $173-201$.

[35] R. Codina, J. Principe, O. Guasch, S. Badia, Time dependent subscsales in the stabilized finite element approximation of incompressible flow problems, Comput. Methods Appl. Mech. Engrg.196(21-24) (2007) 2413-2430. 
[36] O. Guasch, R. Codina, Statistical behavior of the orthogonal subgrid scale stabilization terms in the finite element large eddy simulation of turbulent flows, Comput. Methods Appl. Mech. Engrg.261 (2013) 154-166.

[37] T. Hughes, G. Feijóo, L. Mazzei, J. Quincy, The variational multiscale method, a paradigm for computational mechanics, Comput. Methods Appl. Mech. Engrg.166 (1998) 3-24.

[38] F. Q. Hu, Development of PML absorbing boundary conditions for computational aeroacoustics: A progress review, Comput. Fluids37 (4) (2008) 336-348.

[39] B. Kaltenbacher, M. Kaltenbacher, I. Sim, A modified and stable version of a perfectly matched layer technique for the 3-d second order wave equation in time domain with an application to aeroacoustics, J. Comput. Phys.235 (2013) 407-422.

[40] M. Arnela, O. Guasch, Finite element computation of elliptical vocal tract impedances using the two-microphone transfer function method, J. Acoust. Soc. Am.133(6) (2013) 4197-4209.

[41] V. Allampalli, R. Hixon, M. Nallasamy, S. D. Sawyer, High-accuracy large-step explicit Runge-Kutta (hale-rk) schemes for computational aeroacoustics, J. Comput. Phys.228 (10) (2009) $3837-3850$.

[42] L. Liu, X. Li, F. Q. Hu, Nonuniform-time-step explicit Runge-Kutta scheme for high-order finite difference method, Comput. Fluids105 (0) (2014) $166-178$.

[43] P. Martínez-Lera, C. Schram, Correction techniques for the truncation of the source field in acoustic analogies, J. Acoust. Soc. Am.124 (6) (2008) 3421-3429.

[44] O. Labbé, C. Peyret, G. Rahier, M. Huet, A CFD/CAA coupling method applied to jet noise prediction, Comput. Fluids86 (0) (2013) 1 - 13.

[45] G. Cunha, S. Redonnet, On the signal degradation induced by the interpolation and the sampling rate reduction in aeroacoustics hybrid methods, Int. J. Numer. Meth. Fluids71 (7) (2013) 910-929.

[46] P. Drazin, Introduction to Hydrodynamic Stability, Cambridge Texts in Applied Mathematics, Cambridge University Press, 2002.

[47] M. S. Howe, Acoustics of fluid-structure interactions, Cambridge university press, 1998.

[48] J. Cisonni, K. Nozaki, A. Van Hirtum, X. Grandchamp, S. Wada, Numerical simulation of the influence of the orifice aperture on the flow around a teeth-shaped obstacle, Fluid Dynamics Research 45 (2) (2013) 025505.

[49] R. Hill, J. Wilczak, Pressure structure functions and spectra for locally isotropic turbulence, J. Fluid Mech.296 (1995) $247-269$.

[50] S. Pope, Turbulent Flows, Cambridge University Press, 2000.

[51] M. Howe, R. McGowan, Aeroacoustics of [s], Proc. R. Soc. A461 (2005) 1005-1028.

[52] S. Nittrouer, M. Studdert-Kennedy, R. S. McGowan, The emergence of phonetic segmentsevidence from the spectral structure of fricative-vowel syllables spoken by children and adults, J. Speech Lang. Hear. Res. 32 (1) (1989) 120-132. 